\title{
Сравнительный анализ обратноосмотических мембран методом лазерной десорбции/ионизации
}

\author{
(C) 2021 Шолохова А.Ю., Буряк А.К. \\ Институт физической химии и электрохимии им. А.Н. Фрумкина РАН, Москва \\ Поступила в редакцию 11.05.2021 г.
}

DOI: $10.17308 /$ sorpchrom.2021.21/3627

За последние несколько лет доля рынка обратного осмоса (OO) продолжает расти. ОО имеет тенденцию быть наиболее предпочтительным методом обессоливания вод, поскольку он прост и требует относительно небольшого удельного энергопотребления; поэтому более половины всех установленных опреснительных установок в мире сегодня используют данную технологию. Коммерческие мембраны для ОО обычно состоят из активного слоя ароматического полиамида с микропористым поддерживающим слоем из полисульфона. Но такие мембраны легко подвергаются биологическому отравлению, что ограничивает их применение в очистке воды. При этом также важен контроль поддерживающего слоя для предотвращения его деградации. Целью настоящей работы являлся сравнительный анализ обратноосмотических мембран методом масс-спектрометрии с лазерной десорбцией/ионизацией (ЛДИ), позволяющий выявить сходство и различия в привитых функциональных группах и стабильности полимерных слоев, составляющих мембрану. Масс-спектрометрия ЛДИ представляет собой метод мягкой ионизации с минимальной фрагментацией аналита. Простота, быстрый анализ, высокая производительность, малые объемы аналитов, а также отсутствие или незначительная фрагментация является основными характеристиками ЛДИ-масс-спектрометрии. В работе исследованы четыре образца обратноосмотических мембран (Владимир, АО «РМ Нанотех») и один зарубежный образец. Масс-спектры исследуемых материалов регистрировали на приборе Ultraflex фирмы Bruker, оборудованным азотным лазером с длиной волны 337 нм и энергией 110 мкДж. Запись спектров производили в режиме регистрации положительных ионов в диапазоне $m / z$ 20-2000 Да. В работе показана возможность идентификации пиков характерных для полисульфонового и полиамидного слоев полимерных материалов, проведена идентификация функциональных групп, привитых к полиамидному слою ОО-мембран, определены кластеры неорганических солей на поверхности ОО-мембран, образующиеся в процессе эксплуатации, а также показано их сходство и различие для исследованных мембран. Таким образом, показана возможность быстрого анализа ОО-мембран методом ЛДИ-массспектрометрии. Данный метод позволяет определять компоненты поддерживающего и разделяющего слоев ОО-мембран и образующиеся кластеры неорганических солей на поверхности полимерных материалов.

Ключевые слова: обратноосмотические мембраны, масс-спектрометрия, лазерная десорбция/ионизация, идентификация.

\section{Введение}

Лазерный источник ионов был открыт в начале 60-х годов прошлого века и практически сразу использовался для масс-спектрометрии (МС) [1]. Образование ионов из атомов и молекул путем прямого облучения образца лазерным лучом позже стало известно как лазерная десорбция/ионизация (LDI - Laser Desorption Ionization, ЛДИ - лазерная десорбция/ионизация)[2]. Масс-спектрометрия с лазерной десорбцией/ионизацией представляет собой метод мягкой ионизации с минимальной фрагментацией аналита [3]. Простота, быстрый анализ, высокая производительность, малые объемы аналитов, а также отсутствие или незначительная фрагментация явля- 
ется основными характеристиками ЛДИ [4]. На протяжении многих лет ЛДИ-МС является универсальным аналитическим инструментом для различных приложений, которые варьируются от обнаружения макромолекул, таких как белки и небольшие молекулы, до протеомных исследований [5].

Среди методов ЛДИ, массспектрометрия с ионизацией методом MALDI (Matrix assisted laser desorption/ionization, МАЛДИ - инициированная матрицей лазерная десорбция/ионизация) широко используется при анализе биологических образцов в микробиологии, метаболомике и др. [6]. МАЛДИ, однако, имеет некоторые недостатки, такие как отсутствие универсальной матрицы и образование «горячих точек» во время процесса кристаллизации органической матрицы [7]. Один из методов ЛДИ, разработанный без использования органической матрицы - это ПАЛДИ (SALDI - Surfaceassisted laser desorption/ionization, ПАЛДИ - инициированная поверхностью лазерная десорбция/ионизация) [8]. ПАЛДИ не требует какой-либо органической матрицы и, следовательно, не производит мешающие шумовые ионы в области малых масс, также имеет дополнительное преимущество легкости пробоподготовки и отсутствия «Горячих точек» в подготовленных пробах [9]. ЛДИ-метод без использования органической матрицы был разработан Tanaka et al. [10]. Авторы использовали наночастицы кобальта (30 нм), смешанные с глицерином, для ионизации белков и синтетических полимеров.

Применение масс-спектрометрии для анализа полимерных материалов быстро расширилось с момента разработки МАЛДИ-МС [11,12]. МАЛДИ был применен для анализа биополимеров средней молекулярной массы $[13,14]$ и синтетических полимеров $[15,16]$. Кроме того, полимерные материалы были проанализированы для определения состава мономеров и структуры синтетических полимеров $[17,18]$.

За последние несколько лет доля рынка обратного осмоса (OO) продолжает расти [19]. ОО имеет тенденцию быть наиболее предпочтительным методом обессоливания воды, поскольку он прост и требует относительно небольшого удельного энергопотребления; поэтому более половины всех установленных опреснительных установок в мире сегодня используют технологию ОО [20]. Коммерческие мембраны для ОО обычно состоят из активного слоя ароматического полиамида с микропористым поддерживающим слоем из полисульфона. Но такие мембраны легко подвергаются биологическому отравлению, что ограничивает их применения в очистке воды [21]. При этом также важен контроль поддерживающего слоя для предотвращения его деградации. Целью настоящей работы являлся сравнительный анализ обратноосмотических мембран методом масс-спектрометрии-ЛДИ, позволяющий выявить сходство и различие в привитых функциональных группах и стабильности полимерных слоев, составляющих мембрану.

\section{Экспериментальная часть}

В работе исследованы четыре образца обратноосмотических мембран (Владимир, АО «РМ Нанотех») и один зарубежный образец (табл. 1). Данные образцы являлись экспериментальными и не работали в процессе водоподготовки.

В соответствии с технологией производителя для сохранения эксплуатационных характеристик и предотвращения микробиологического воздействия «мокрые» мембранные элементы консервируются раствором, содержащим $1 \%$ метабисульфита натрия. Для анализа «мокрые» мембранные элементы были отмыты деионизованной водой, высушены при комнатной температуре и было проведено их механическое разделение на отдельные слои. 
Таблица 1. Характеристики мембран, исследуемых в работе

Table 1. Characteristics of membranes studied in the work

\begin{tabular}{|c|c|c|}
\hline Мембрана & Слои & Состав \\
\hline \multirow{3}{*}{ Мембрана 1} & Субстрат & полиэтилентерефталат \\
\hline & $\begin{array}{l}\text { Поддерживающий } \\
\text { слой }\end{array}$ & $\begin{array}{c}\text { полисульфон, полиэфирсульфон, диметилформамид, } \\
\text { диметилацетамид }\end{array}$ \\
\hline & $\begin{array}{l}\text { Разделяющий } \\
\text { слой }\end{array}$ & $\begin{array}{c}\text { изофталоилхлорид, метафенилендиамин, модификатор } \\
\text { на основе карбодиимидов }\end{array}$ \\
\hline \multirow{3}{*}{ Мембрана 2} & Субстрат & полиэтилентерефталат \\
\hline & $\begin{array}{l}\text { Поддерживающий } \\
\text { слой }\end{array}$ & $\begin{array}{c}\text { полисульфон, полиэфирсульфон, диметилформамид, } \\
\text { диметилацетамид }\end{array}$ \\
\hline & $\begin{array}{l}\text { Разделяющий } \\
\text { слой }\end{array}$ & изофталоилхлорид, метафенилендиамин \\
\hline \multirow{3}{*}{ Мембрана 3} & Субстрат & полиэтилентерефталат \\
\hline & $\begin{array}{c}\text { Поддерживающий } \\
\text { слой } \\
\end{array}$ & полисульфон, диметилформамид \\
\hline & $\begin{array}{l}\text { Разделяющий } \\
\text { слой }\end{array}$ & $\begin{array}{l}\text { изофталоилхлорид, метафенилендиамин, } \\
\text { (реагент 553) - (3-аминопропил)силантриол }\end{array}$ \\
\hline \multirow{3}{*}{ Мембрана 4} & Субстрат & полиэтилентерефталат \\
\hline & $\begin{array}{l}\text { Поддерживающий } \\
\text { слой }\end{array}$ & полисульфон, диметилформамид \\
\hline & $\begin{array}{l}\text { Разделяющий } \\
\text { слой }\end{array}$ & $\begin{array}{c}\text { изофталоилхлорид, тримезоилхлорид, метафенилен- } \\
\text { диамин, фторсодержащий аминосилан }\end{array}$ \\
\hline \multirow{3}{*}{$\begin{array}{c}\text { Мембрана } 5 \\
\text { (зарубежный } \\
\text { образец) }\end{array}$} & Субстрат & полиэтилентерефталат \\
\hline & $\begin{array}{l}\text { Поддерживающий } \\
\text { слой }\end{array}$ & полисульфон, диметилформамид \\
\hline & $\begin{array}{l}\text { Разделяющий } \\
\text { слой }\end{array}$ & $\begin{array}{c}\text { изофталоилхлорид, четвертичные аммониевые } \\
\text { основания }\end{array}$ \\
\hline
\end{tabular}

ЛДИ-МС. Масс-спектры исследуемых материалов регистрировали на приборе Ultraflex фирмы Bruker, оборудованном азотным лазером с длиной волны 337 нм и энергией 110 мкДж. Масс-анализатор времяпролетный. Запись спектров производили в режиме регистрации положительных ионов в диапазоне $\mathrm{m} / \mathrm{z} 20$ 2000 Да при энергии лазера 30-95\% от максимальной. Режим работы лазера: 2550 импульсов с частотой 20-50 Гц.

\section{Обсуждение результатов}

Проведен анализ обратноосмотических мембран методом лазерной десорбции/ионизации в режиме регистрации положительных ионов. На рисунке 1 представлен масс-спектр мембраны 1.

Масс-спектр мембраны 1 содержит фрагменты полисульфоновой матрицы, масс-спектрометрические сигналы которой соответствуют $\mathrm{m} / z$ 134, 214, 227.
Данные фрагменты являются типичными для мембран, имеющих полисульфоновую подложку, пики, соответствующие данным фрагментам, наблюдаются для всех исследуемых образцов [23].

Также наблюдается ряд пиков, соответствующих кластерам ионов натрия и калия с сульфогруппой. В таблице 2 представлено сравнение измеренного и теоретического отношения массы к заряду.

Следует отметить, что в массспектрах, полученных для других образцов мембран, наблюдаются такие же пики. Также наблюдается ряд пиков с $\mathrm{m} / z$ 81, 98, соответствующих кластерам ионов натрия и калия с хлоридом, а именно $\mathrm{Na}[\mathrm{NaCl}]$ и $\mathrm{Na}[\mathrm{KCl}]$, соответственно. Для образца мембраны 2 также наблюдается пик $m / z 114-\mathrm{K}[\mathrm{KCl}]$, а для образца мембраны 4 также наблюдается пик $m / z 172-\mathrm{Na}\left[\mathrm{K}_{2} \mathrm{Cl}_{2}\right]$. 


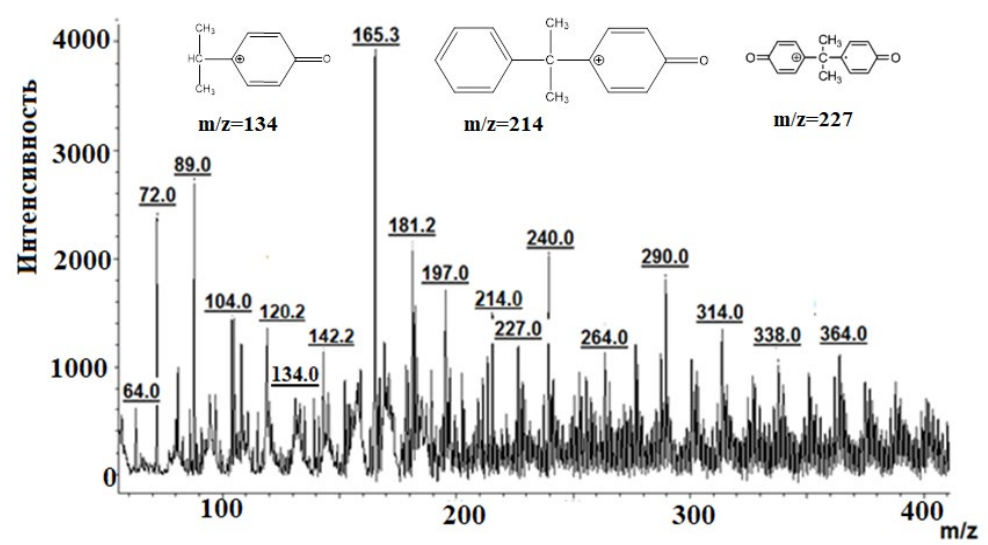

Рис. 1 Масс-спектр мембраны 1 в режиме регистрации положительных ионов

Fig. 1. Mass spectrum of membrane 1 in the mode of registering positive ions

Компонентами разделяющего слоя мембраны являются фенилендиамин и тримезоилхлорид, межфазная полимеризация между которыми протекает согласно схеме [24]:

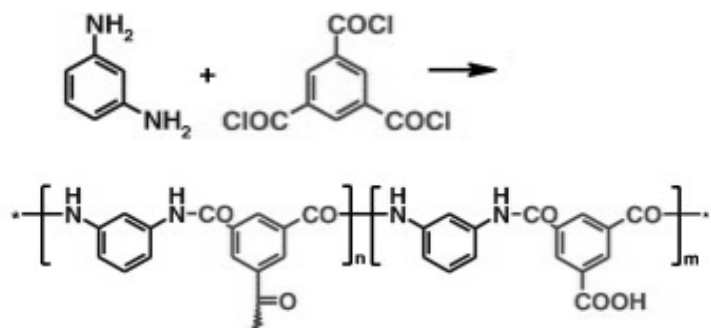

Продуктам межфазной поликонденсации метафенилендиамина с трифталоилхлоридом соответствуют фрагменты с $\mathrm{m} / \mathrm{z} 264.0$ и $\mathrm{m} / \mathrm{z} 280.0$ :

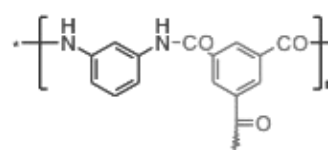<smiles>CNc1cccc(NC(=O)c2cccc(C(=O)O)c2)c1</smiles>

$\mathrm{m} / \mathrm{z} 264.0$ $m / z 280.0$

Наличие функциональной группы (1-Этил-3-(3-диметиламинопропил)карбодиимид, EDC) привитой к полиамид- ному слою подтверждается пиком с $\mathrm{m} / z$ 290.0. Образование данного продукта протекает по следующей схеме [25]:

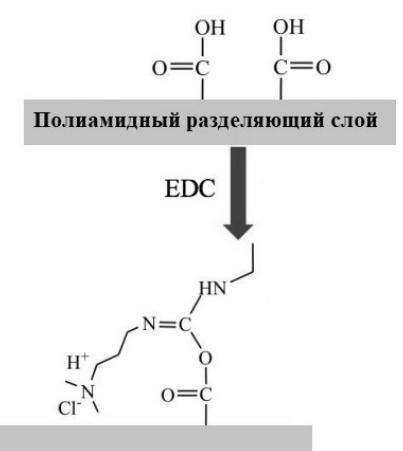

Анализ мембраны 1, выполненный методом ЛДИ-масс-спектрометрии, показал наличие фрагментов полисульфона, полиамида и привитого в качестве функциональных групп, предположительно, (1-Этил-3-(3-диметиламинопропил)карбодиимида.

Анализ всех образцов ОО-мембран также показал наличие фрагментов полисульфона и привитых функциональных групп (табл.3).

Таблица 2. Кластерные ионы, полученные при ионизации сульфогрупп с поверхности мембраны

Table 2. Cluster ions obtained by ionisation of sulfogroup on the surface of the membrane

\begin{tabular}{|c|c|c|}
\hline Ион & $m / z$ тeop. & $m / z$ эксп. \\
\hline $\mathrm{SO}_{2}{ }^{-}$ & 64.06 & 64.0 \\
\hline $\mathrm{Na}\left[\mathrm{H}_{2} \mathrm{SO}_{2}\right]$ & 89.07 & 89.0 \\
\hline $\mathrm{Na}\left[\mathrm{HSO}_{3}\right]$ & 104.06 & 104.0 \\
\hline $\mathrm{K}\left[\mathrm{HSO}_{3}\right]$ & 120.17 & 120.2 \\
\hline $\mathrm{K}\left[\mathrm{NaSO}_{3}\right]$ & 142.15 & 142.2 \\
\hline $\mathrm{Na}\left[\mathrm{Na}_{2} \mathrm{SO}_{4}\right]$ & 165.03 & 165.3 \\
\hline $\mathrm{Na}_{2}\left[\mathrm{KSO}_{4}\right]$ & 181.14 & 181.2 \\
\hline $\mathrm{Na}\left[\mathrm{K}_{2} \mathrm{SO}_{4}\right]$ & 197.25 & 197.0 \\
\hline
\end{tabular}


Таблица 3. Сравнение мембран, режим регистрации положительных ионов

Table 3. Comparison of membranes, the mode of registering positive ions

\begin{tabular}{|c|c|c|c|c|}
\hline Мембрана 1 & Мембрана 2 & Мембрана 3 & Мембрана 4 & Мембрана 5 \\
\hline & & $134^{*}$ & & $134^{*}$ \\
\hline & & $172^{*}$ & & \\
\hline $214^{*}$ & $214^{*}$ & $214^{*}$ & $214^{*}$ & $214^{* *}$ \\
\hline $227^{*}$ & & & & \\
\hline & & & $242^{* *}$ & \\
\hline & & $258^{* *}$ & & \\
\hline & & $278^{* *}$ & & \\
\hline $290^{* *}$ & & & & \\
\hline & $403^{* *}$ & & & \\
\hline
\end{tabular}

*фрагменты полисульфоновой матрицы; **фрагменты, соответствующие функциональным группам

*fragments of the polysulphone matrix; **fragments corresponding to functional groups

В образце мембраны 2 наличие функциональной группы (3-аминопропил)силантриол), привитой к полиамидному слою подтверждается пиком с $m / z$ 403. В образце мембраны 3 наличие привитого в качестве функциональной группы, предположительно, фторсодержащего аминосилана, подтверждается пиком с $m / z$ 242, соответствующим фрагменту фторсодержащего аминосилана и карбоксильной группы матрицы. Наличие функциональной группы (содержащей фторсодержащие фрагменты), привитой к полиамидному слою подтверждается пиками с $\mathrm{m} / \mathrm{z} 258.0$ и 278.1. Разница в $\mathrm{m} / z 20$ (HF), что, предположительно указывает на наличие фторсодержащих фрагметов.

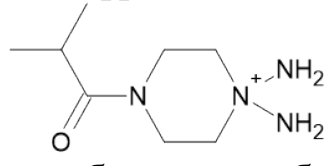

Масс-спектр образца мембраны 5 содержит пик с $m / z 185.0$, который предположительно соответствует следующему соединению:

Таким образом, показана возможность быстрого анализа ОО-мембран ме-

\section{Список литературы}

1. Honig R.E., Woolston J.R. // Applied Physics Letters. 1963. Vol. 2. No 2. pp. 138139. doi:10.1063/1.1753812.

2. Zakett D., Schoen A.E., Cooks R.G., Hemberger P.H. // Journal of the American Chemical Society. 1981. Vol. 103. No 5. pp. тодом ЛДИ-масс-спектрометрии. Данный метод позволяет определять компоненты поддерживающего и разделяющего слоев ОО-мембран и образующиеся кластеры неорганических солей на поверхности полимерных материалов.

\section{Заключение}

В результате сравнительного исследования пяти образцов обратноосмотических мембран методом ЛДИ-массспектрометрии показано, что данный метод дает возможность проводить быстрый и информативный анализ ООмембран. Метод позволяет определять компоненты поддерживающего и разделяющего слоев и идентифицировать функциональные группы, привитые к полиамидному слою ОО-мембран. Обнаружены и идентифицированы кластеры неорганических солей на поверхности ОО-мембран и показано их сходство и различие для разных мембран.

1295-1297. doi:10.1021/ja00395a086.

3. Arakawa R., Kawasaki H. // Analytical Sciences. 2010. Vol. 26. No 12, pp. 1229-1240. doi:10.2116/analsci.26.1229.

4. Bergman N., Shevchenko D. // Analytical and Bioanalytical Chemistry. 2014. Vol. 406. No 1. pp. 49-61. doi:10.1007/s00216-013-7471-3.

5. Mandal A., Singha M., Addy P.S., Basak 
A. //Mass Spectrometry Reviews. 2019. Vol. 38. No 1. pp. 3-21. doi:10.1002/mas.21545.

6. El-Aneed A., Cohen A., Banoub J.M. // Applied Spectroscopy Reviews. 2009. Vol. 44. No 3. pp. 210-230. doi:10.1080/0570492 0902717872 .

7. Krutchinsky A.N., Chait B.T. // Journal of the American Society for Mass Spectrometry. 2002. Vol. 13. No 2. pp. 129-134. doi:10.1016/ S1044-0305(01)00336-1.

8. Dattelbaum A.M., Iyer S. // Expert Rev. Proteomics. 2006. Vol. 3. No 1. pp. 153-161. doi:10.1586/14789450.3.1.153.

9. Song K., Cheng Q. // Applied Spectroscopy Reviews. 2019. Vol. 55. No 13. pp. 1-23. doi:10.1080/05704928.2019.1570519.

10.Tanaka K., Waki H., Ido Y., Akita S. et al., $1 /$ Rapid Communications in Mass Spectrometry. 1988. Vol. 2. No 8. pp. 151-153. doi:10.1002/rcm.1290020802.

11.Kang M.J., Seong Y., Kim M.J., Kim M.S. et al. // Applied Chemistry for Engineering. 2017. Vol. 28. No 3. pp. 263-271. doi:10.14478/ace.2017.1019.

12.Пыцкий И.С., Кузнецова Е.С., Буряк А.К. // Сорбционные и хроматографические nрочессы. 2021. Т. 21. № 1. С. 69-76. doi: https://doi.org/10.17308/sorpchrom.2021.21/32 21

13.López-García M., García M.S.D., Vilariño J.M.L., Rodríguez M.V.G. // Food Chemistry. 2016. Vol. 199. pp. 597-604. doi:10.1016/ j.foodchem.2015.12.016.

14.Kosyakov D.S., Anikeenko E.A., Ul'yanovskii N. V., Khoroshev O.Y. et al. // Analytical and Bioanalytical Chemistry. 2018. Vol. 410. No 28. pp. 7429-7439. doi:10.1007 s00216-018-1353-7.

15.Spring A.M., Maeda D., Ozawa M., Odoi
K. et al. // Polymer. 2015. Vol. 56. pp. 189-198. doi:10.1016/j.polymer.2014.11.043.

16.Gies A.P., Ellison S.T., Stow S.M., Hercules D.M. // Analytica Chimica Acta. 2014. Vol. 808. pp. 124-143. doi:10.1016/ j.aca.2013.09.007.

17.Nishimori K., Ouchi M., Sawamoto M. // Macromolecular rapid communications. 2016. Vol. 37. No 17. pp. 1414-1420. doi:10.1002/ marc.201600251.

18.Yol A.M., Dabney D.E., Wang S.-F., Laurent B.A. et al. // J. Am. Soc. Mass Spectrom. 2013. Vol. 24. No 1. pp. 74-82. doi:10.1007/s13361-012-0497-5.

19. Giwa A., Akther N., Dufour V., Hasan S.W. // RSC Advances. 2016. Vol. 6. No 10. pp. 8134-8163. doi:10.1039/c5ra17221g.

20.Shenvi S.S., Isloor A.M., Ismail A.F. // Desalination. 2015. Vol. 368. pp. 10-26. doi:10.1016/j.desal.2014.12.042.

21.Kang G.D., Gao C.J., Chen W.D., Jie X.M., et al. // Journal of Membrane Science. 2007. Vol. 300. No 1-2. pp. 165-171. doi:10.1016/j.memsci.2007.05.025.

22.ГОСТ 10896-78 : Иониты. Подготовка к испытанию. Введен 1980-01-01. Москва : Издательство стандартов, 1980.

23.Ellison S.T., Gies A.P., Hercules D.M., Morgan S.L. // Macromolecules. 2009. Vol. 42. No 8. pp. 3005-3013. doi:10.1021/ma900161y.

24.Lau W.J., Ismail A.F., Misdan N., Kassim M.A. // Desalination. 2012. Vol. 287, pp. 190199. doi:10.1016/j.desal.2011.04.004.

25.Liu M., Yu C., Wu Y., Lü Z. et al. // Chemical Engineering Research and Design. 2019. Vol. 141. pp. 402-412. doi:10.1016/ j.cherd.2018.11.009.

\title{
A comparative analysis of reverse osmosis membranes by laser desorption/ionisation
}

\author{
(C) 2021 Sholokhova Yu.A., Buryak A.K. \\ Frumkin Institute of Physical Chemistry and Electrochemistry of the Russian Academy of Sciences, Moscow
}

The market share for reverse osmosis (RO) has been growing over the past few years. The popularity of the RO can be explained by its simplicity and a relatively low energy intensity. As a result, this technology is being used by more than half desalination units installed all over the world. Commercial RO membranes typically consist of an active layer of an aromatic polyamide with a microporous polysulphone support layer. However, such membranes are easily exposed to biological fouling, which limits their use in water purification. It is also important to control their support layer to prevent its degradation. The aim of this work was to conduct a comparative analysis of reverse osmosis membranes by laser desorption/ionisation (LDI), 
which made it possible to reveal similarities and differences between grafted functional groups and compare the stability of the polymer layers that make up the membrane. Mass spectrometry is a method of soft ionisation with minimum analyte fragmentation. The main features of the LDI involve its simplicity, possibility of fast analysis, high productivity, low volumes of analytes, and no or low fragmentation. Four samples of reverse osmosis membranes (Vladimir, AO RM Nanotek) and one sample representing their foreign analogue were studied in this work. The mass spectra of the studied materials were recorded on a Bruker Ultraflex device equipped with a nitrogen laser with a wavelength of $337 \mathrm{~nm}$ and the energy of $110 \mu \mathrm{J}$. The spectra were recorded in the mode of registering positive ions within the range of $m / z 20-2.000 \mathrm{Da}$. The paper shows the possibility of identifying peaks characteristic of the polysulphone and polyamide layers in polymeric materials, identifies the functional groups grafted onto the polyamide layer of RO membranes, identifies clusters of inorganic salts on the surface of RO membranes formed during the operation, and shows their similarities and differences in the studied membranes. Therefore, the research showed the possibility of performing a rapid analysis of RO membranes by the method of LDI mass spectrometry. This method makes it possible to determine the components of the support and separation layers of RO membranes and the clusters of inorganic salts formed on the surface of polymeric materials.

Keywords: reverse osmosis membranes, mass spectrometry, laser desorption/ionisation, identification.

\section{References}

1. Honig R.E., Woolston J.R., Applied Physics Letters, 1963, Vol. 2, No 2, pp. 138-139. doi:10.1063/1.1753812.

2. Zakett D., Schoen A.E., Cooks R.G., Hemberger P.H., Journal of the American Chemical Society, 1981, Vol. 103, No 5, pp. 1295-1297. doi:10.1021/ja00395a086.

3. Arakawa R., Kawasaki H., Analytical Sciences, 2010, Vol. 26, No 12, pp. 1229-1240. doi:10.2116/analsci.26.1229.

4. Bergman N., Shevchenko D., Analytical and Bioanalytical Chemistry, 2014, Vol. 406, No 1, pp. 49-61. doi:10.1007/s00216-013-74713.

5. Mandal A., Singha M., Addy P.S., Basak A., Mass Spectrometry Reviews, 2019, Vol. 38, No 1, pp. 3-21. doi:10.1002/mas.21545.

6. El-Aneed A., Cohen A., Banoub J.M., Applied Spectroscopy Reviews, 2009, Vol. 44, No 3, pp. 210-230. doi:10.1080/0570492090271 7872 .

7. Krutchinsky A.N., Chait B. J., Am. Soc. Mass Spectrom., 2002, Vol. 13, No 2, pp. 129134. doi:10.1016/S1044-0305(01)00336-1.

8. Dattelbaum A.M., Iyer S., Expert Rev. Proteomics, 2006, Vol. 3, No 1, pp. 153-161. doi:10.1586/14789450.3.1.153.

9. Song K., Cheng Q., Applied Spectroscopy Reviews, 2019, Vol. 55, No 13, pp. 1-23. doi:10.1080/05704928.2019.1570519.

10. Tanaka K., Waki H., Ido Y., Akita S. et al., Rapid Communications in Mass Spectrometry, 1988, Vol. 2, No 8, pp. 151-153. doi:10.1002/ rcm.1290020802.

11.Kang M.J., Seong Y., Kim M.J., Kim M.S., et al., Applied Chemistry for Engineering, 2017,
Vol. 28, No 3, pp. 263-271. doi:10.14478/ ace.2017.1019.

12.Pyckij I.S., Kuznecova E.S., Burjak A.K., Sorbtsionnye i khromatograficheskie protsessy, 2021, Vol. 21, No 1, pp. 69-76. doi: https://doi.org/10.17308/sorpchrom.2021.21/32 21

13.López-García M., García M.S.D., Vilariño J.M.L., Rodríguez M.V.G., Food Chemistry, 2016, Vol. 199, pp. 597-604. doi:10.1016/j.foodchem.2015.12.016.

14.Kosyakov D.S., Anikeenko E.A., Ul'yanovskii N.V., Khoroshev O.Y. et al., Analytical and Bioanalytical Chemistry, 2018, Vol. 410, No 28, pp. 7429-7439. doi:10.1007/ s00216-018-1353-7.

15.Spring A.M., Maeda D., Ozawa M., Odoi K. et al., Polymer, 2015, Vol. 56, pp. 189-198. doi:10.1016/j.polymer.2014.11.043.

16.Gies A.P., Ellison S.T., Stow S.M., Hercules D.M., Analytica Chimica Acta, 2014, Vol. 808, pp. 124-143. doi:10.1016/ j.aca.2013.09.007.

17.Nishimori K., Ouchi M., Sawamoto M., Macromolecular rapid communications, 2016, Vol. 37, No 17, pp. 1414-1420. doi:10.1002/ marc.201600251.

18. Yol A.M., Dabney D.E., Wang S.-F., Laurent B.A. et al., ,J. Am. Soc. Mass Spectrom., 2013, Vol. 24, No 1, pp. 74-82. doi:10.1007/ s13361-012-0497-5.

19. Giwa A., Akther N., Dufour V., Hasan S.W., RSC Advances, 2016, Vol. 6, No 10, pp. 8134-8163. doi:10.1039/c5ra17221g.

20.Shenvi S.S., Isloor A.M., Ismail A.F., Desalination, 2015, Vol. 368, pp. 10-26. doi:10.1016/j.desal.2014.12.042. 
21.Kang G.D., Gao C.J., Chen W.D., Jie X.M. et al., Journal of Membrane Science, 2007, Vol. 300, No 1-2, pp. 165-171. doi:10.1016/ j.memsci.2007.05.025.

22.GOST 10896-78: Ionity. Podgotovka k ispytaniju. - Vveden 1980-01-01. M., Izdatel'stvo standartov, 1980.

23.Ellison S.T., Gies A.P., Hercules D.M., Morgan S.L., Macromolecules, 2009, Vol. 42, No 8, pp. 3005-3013. doi:10.1021/ma900161y.

Шолохова Анастасия Юрьевна - к.х.н., н.с. лаборатории физико-химических основ хроматографии и хромато-масс-спектрометрии, Институт физической химии и электрохимии имени А.Н. Фрумкина РАН, Москва

Буряк Алексей Константинович - заведующий лабораторией физико-химических основ хроматографии и хромато-масс-спектрометрии, проф, д.Х.н. Институт физической химии и электрохимии имени А.Н. Фрумкина РАН, Москва
24.Lau W.J., Ismail A.F., Misdan N., Kassim M.A., Desalination, 2012, Vol. 287, pp. 190199. doi:10.1016/j.desal.2011.04.004.

25.Liu M., Yu C., Wu Y., Lü Z. et al., Chemical Engineering Research and Design, 2019, Vol. 141, pp. 402-412. doi:10.1016/ j.cherd.2018.11.009.

Sholokhova Anastasiya Yu. - PhD, researcher, laboratory of physicochemical principles of chromatography and chromatography - mass spectrometry; Institute of Physical Chemistry and Electrochemistry, Moscow, e-mail: shonastya@yandex.ru

Buryak Alexey K. - prof., grand $\mathrm{PhD}$ (chemistry), laboratory of physicochemical principles of chromatography and chromatography - mass spectrometry; Institute of Physical Chemistry and Electrochemistry, Moscow, e-mail: akburyak@mail.ru 